\title{
Archaeological Databases and GIS: Working with Databases
}

\author{
Thomas Mafredas and George Malaperdas
}

\section{ABSTRACT}

Digital databases are considered nowadays, necessary for the organization of a recent archeological project. Typically, one of the main issues at the stage of archaeological surface research preparation is the method of recording all the archaeological information that will emerge, which is directly dependent on two factors, the difference of each area in terms of its geomorphology, including the climate and general environmental conditions, and the different approach to the objectives to be achieved by the leading archaeologists. As a consequence of all of this, there is no such thing as a uniquely generated form that can act as a guideline. This paper provides some basic database knowledge as well as a case study with a database example.

Published Online: June 21, 2021

ISSN:2736-5492

DOI : $10.24018 /$ ejcompute.2021.1.3.20

Thomas Mafredas

(e-mail:mafredas@ gmail.com)

George Malaperdas

(e-mail: envcart@yahoo.gr)

*Corresponding Author

Keywords: Archeological database, databases, DBMS, GIS.

\section{INTRODUCTION}

Databases are a functional tool for depicting various facts of reality by enumerating entities and concepts with the help of computer technology. They are a coherent collection of data of intrinsic importance and uniform structure and designed to host data for a particular purpose. Their construction is intended for specific users and applications, and it is easy to understand and accessible to everyone. Databases can be stored either as files or in specialized programs called Database Management Systems (DBMS) [1],[2].

The Database Management System is a system for creating, processing maintaining and developing the Database which makes the data management procedures much easier. This system initially sets the database, i.e., the specifications of the types, structures and limitations of the data to be stored. All the features of a database are defined based on its creation purpose and the kind of information which wanted to be extracted from the application. Next is the construction of the database, namely the storage of the data and the setting of all these questions for extracting specific data. In other words, the structure of a database is consisting of a data model, tools set and relations for the describing and interpreting the data meaning of [1].

The DBMS are designed to provide reliable and secure means of storing data, straightforward means of retrieving data, and safe means of modifying stored data. They exist solely to ease the task of accessing and managing often large and complex collections of functionally related data or databases. In this point, it should be noticed that, unlike many other types of software systems, DBMS do very little of direct concern to an end-user whose main interest is more often an archaeological task or problem. Nevertheless, much of the information presented and analyzed within a modern GIS, are presented on the Web or visualized in a VR system may well have been derived from a database. Similarly, archives and monument registers are dependent on DBMS for the management of their data holdings. DBMS can be seen as a technology that underpins and supports many aspects of archaeological computing, rather than a tool for performing archaeological tasks [3].

So, a DBMS is a software application designed to organize data storage and access in an efficient and effective way. Among the main capabilities provided by DBMS are the following:

- A data model, a mechanism for the digital representation of real-world objects.

- The ability to import data into the database through tools designed for this purpose.

- Indexes, mechanisms that increase the rapidity of the search.

- Language query formulation, a standard method about queries syntax and use commands to the database.

- Security, controlled access to the data by the users.

- Controlled update, which ensures the organized implementation of updates to the data from multiple users at once

- Backups, with tools that store with security and can recovery parts or entire database.

- Management tools designed to cover the needs from the beginning, designing the database up to managing users and indexes or configuring performance parameters.

- Applications, standard tools to facilitate the creation and maintenance of databases.

- Application programming interfaces (APIs), which allow additional functionality to be added [1], [2]. 


\section{Methodology - Archaeological Database And GIS}

The application of GIS in Archaeology has provided significant benefits in organizing and analyzing the excavations' archive [4],[5]. It is especially successful in the management of spatial information, in the integration of new ways of digital imaging and their analytical capabilities in space-time correlations of archaeological material [6],[7].At the same time, special emphasis was placed on recording the spatial properties of the mobile and immovable excavations' finds. Layout of a topographic grid, which always preceded the excavation, provided the background for organizing logging in the field. The introduction of GIS into the archaeologist's toolkit means that complex, contextual geographical relationships can be more readily quantified than in the past; GIS facilitates the rapid integration and analysis of spatial information, while provide many advantages in data acquisition techniques and computational power, which means that the work is not any more time consuming [8],[9].

The uses of GIS are divided into three classes, and this classification system remains an effective way to discuss the archaeological uses of GIS. The first class calls upon GIS to be used for its traditional purpose, mainly to create maps, becoming "little more than a two-dimensional (2D) cartographic presentation tool" [10]. Initially, many archaeologists predicted that GIS would eventually stagnate and do little more than reproduce the uses of CAD programs. GIS software includes, however, a number of sophisticated features not available in CAD. The ability to link with database management systems allows the user to access information from a visual interface rather than a text-driven one.

The second class begins to draw upon these more advanced uses of GIS to complete complex analysis, rarely undertaken. Examples of this type include the predictive modeling of archaeological sites, which involves bringing together large amounts of data, such as slope and soil type, and examining the relationships that exist among known sites and these resources. The third class of GIS uses looks at new and unique methods of analysis. These techniques include two wholly new concepts; both born out of GIS developments. The first is termed cost surface analysis (CSA) and assigns weights to individual physical locations. The second new technique is termed viewshed analysis and has become one of the most common analytical uses of GIS in archaeological studies of landscape. Viewshed analysis has been used to address the social statements associated with assigned meanings of visible location [9].

From the beginning, one of the first stages was the incorporation of realistic depictions of the archaeological findings of the excavations. Archaeologists are excavating the real space, but they interpret two-dimensional drawings. Interpretation can be greatly enhanced if archaeological evidence is able to be observed in all horizontal and vertical aspects. The data from the systematic recording of archaeological objects can be used in a GIS environment by helping archaeologists to portray realistic objects that have been removed. One of the main problems of the excavation methodology is the dividing of a layer in many other layers which has as result the fragmentation of the excavated information associated with a layer. The proposed methodology for the construction of three-dimensional objects is based on a combination of photogrammetric techniques and the efficient use of specialized geological software (EVS).

The generated objects can be imported into ArcGIS software, where they can be used for visualization and analysis. More specifically, using the program tools can be grouped into excavated sections depending on the layer and then visually collated with the vertical incisions recording the actual stratigraphy. In an analogous way, the excavated sections could be visually examined in conjunction with the plans of the immobile findings in order to verify the correctness of the initial investigation and the better understanding of the stratigraphic correlations to the using surfaces. Thus, we observe that the detailed information recorded in a database for each excavation unit can be combined with the representations in a particular space-time frame. At the same time, the depiction of movable finds as points within the dimensional space allows exploration of their spatial dispersion. The use of appropriate notations can group findings according to their species (e.g., stone tools) and associate them with both the stratigraphy and other objects [8].

As a result of the geodatabases and GIS use in excavation, archaeologists have gained a better understanding of and visualize the distribution, concentrations, and spatial relationships of artifacts to settlement groups that have inhabited to a site from a particular time period to another. Also,it's possible for them to set all the data in chronically sequence. Moreover, they could synthesize and analyze the intra-site data and reassembling past cultural practices in order to draw conclusions about social trends. Furthermore, it is obvious that the researchers could improve the abilities to target critical grids of archaeological interest within each of the stratification layers, and to identify correlations and trends associated with specific periods of inhabited time. This also serves as a tool in establishing direction for future excavation work. Stratigraphic sequences introduce a new level of complexity when compared with traditional GIS visualizations. This is really useful in spatial places which were settled on numerous occasions over the centuries and could offer a unique opportunity to introduce and exploit GIS capabilities [11].

There are numerous advantages of structuring GIS data in a geodatabase environment with some of the most important concern the structure of GIS data itself. Vector data including points, lines, and polygons are typically stored in shape files referred to as feature classes. A geodatabase allows the creation of feature data sets that enable coordinated relationships among feature classes. Another advantage of using a geodatabase structure is that allows the creation of domains. A domain assigns valid values or ranges for the attribute table that forms part of the information contained within a feature class, helping to reduce errors in data entry by eliminating invalid entries. It also reduces data entry time by creating a series of drop-down menus. The construction of a geodatabase similarly has advantages for future research. Since GPS and survey data are recorded in a format directly translated into a GIS file, these files can be incorporated into the geodatabase. Finally, a geodatabase relaxes the system 
requirements needed to run GIS software on a computer. The geodatabase is one system file, and the GIS software, no matter what is asked of it, only has to access one file, freeing up system resources [12].

While the basic structure of the geodatabase is drawn from a wide variety of recording schemes, it cannot address the range of potential research questions that are sometimes formed by researchers. One approach is to record as much information about each artifact and feature as possible. The benefit of using a geodatabase is the ability to assign an unlimited number of attribute columns to each feature class. The most basic element is the feature class or shape file consisting of point, line, or polygon features. Feature classes are grouped together to form feature datasets that can hold an unlimited number of feature classes. This is an important consideration for the research design phase as well. GIS can map every object found in a site, provided the details of recording that has been kept. Alternatively, the inclusion of feature identifications or excavation unit identifications in the artifact spreadsheet allows the archaeologist to minimally visualize which features or units contain which types of artifacts [12].

A useful starting point for a Geodatabase design is to employ a model during the research design phase of archaeological research. Two aspects of geodatabase creation have to be carefully addressed by the designer.

First, how will the geodatabase feature class and feature datasets, be organized?

Second, what kind of data will be included in the attribute tables of each feature class? Most of the geodatabases have started with six datasets, each containing a number of feature classes, and during the use of the database, most of the times, have added some more datasets, always depending of the needs of the archaeological project and geodatabases' users [8], [9], [11], [12].

- Arc Tools: Contains feature classes that represent data specifically related to the archaeological organization of work, such as base datum points, dimensions of the sites (polygons), and feature number annotations (labels).

- Boundaries: Contains polygons that represent survey and site boundaries as established during fieldwork.

- Structures: Contains point, multipoint, line, and polygon files used to represent all objects associated with buildings, whether standing or in ruin.

- Artifacts: Contains point, line, and polygon files representing individual artifacts or scatters of artifacts.

- Environment: Contains information from the natural environment or landscape, including contour data.

- Transportation: Includes point, line, and polygon files representing any type of transportation facility, such as roads and railways, with both historic and modern features included.

Approaching archaeological work with these classifications in mind ensures valuable information is not overlooked or lost. The selection of attribute data for any geodatabase is case-by-case and research design dependent; however, there are common types of information that should be attached to each feature class. These include site name, identification number, occupation data, method of collection, historic context, basic measurements, photographic information, and information for linking to an external database, and similar types of records. It is important to emphasize that ArcGIS and the geodatabase design functions like a typical database management system in that researchers can search and select features by attributes, find features by searching for specific attributes, and generate reports using any of the attribute data [9],[12].

According to what had discussed above the GIS expand upon and create new and superior visualization opportunities in archaeology beyond the traditional section-style drawing, while there have been some scholars had examined in depth the benefits that a geodatabase can bring to site analysis when carefully designed feature sets with properly established relationships and domains are included. Data entry efficiency and accuracy can be greatly improved due to limited choices offered, eliminating invalid entries. Digital cataloging establishes data consistency may serve as a model for other ancient sites in the region with similar artifact find catalogues [11].

Representing excavation data via visualization and illustrative techniques on maps and models could help to gain new insights through spatial analysis. It may be possible to establish patterns through visualization that go undetected otherwise. GIS analytics provide the ability to spatially relate the strata to one another and to visualize materials from each inhabited era while also keeping them in context with one another. Subsequent and previous settlement patterns can also be easily visualized and compared.

Data collection techniques via the use of handheld instruments can facilitate the uniform collection and recording of finds in the field. The data accuracy can be improved in the field by implementing a workflow that includes handheld devices loaded with a data entry form that restricts users to choosing from specific values. This improves the spatial context of the objects and provenience by attaching precise latitude $(\mathrm{x})$ and longitude $(\mathrm{y})$ coordinates at the time of the find [11].

\section{THE BETHSAIDA CASE STUDY}

In this project the main goal was the development of a Database with archaeological data entry and parallel implementation of GIS for capturing these data in a geophysical map. The result was a development of a database that could show the geographical location of the objects found in the excavation.In any case the way that the final user could use the data always depended on the initial queries which synthesized the database, while there was the possibility of evolving and developing the initial queries.

One of the first steps was the development of ARCHEOGIS, a web-based geodatabase application, based on MS-Access; aimed to improve access and entry of excavation data remotely. One of the main problems for this project was the small geographic context, because Bethsaida was a 26-acre parcel, and active grid squares within that area may be only 100 square meters in size. Nowadays the equipment has much greater capacity for accuracy than in the past; the recording finds can be accurate to the nearest centimeter in some cases. Thus, in a very small geographical context there is a lot of ability of GIS using to be precise and thorough in the documentation and handling of archaeological finds and to exploit the depth, or vertical 
nature of time and the law of superposition.

Data collection and entry for the Bethsaida Excavation was vastly improved through the adoption of handheld devices in the field, which allowed the rapid gathering of data and fast processing time for further analysis using new GIS techniques. It was found that although the data collection process was more cumbersome in the field, the reduced postprocessing time greatly improved productivity and workflow efficiency, citing increased accuracy of point placement within the excavation survey area, which was favorable to visualizing a general or random distribution of point density. These accurately were illustrated and replicated actual artifact concentrations.

One of the main problems for developing the database was the entry of the data. It is obvious that inconsistent data entry presents numerous problems in analysis and query results. It was found that handheld data capture revolutionized the ability to quantify spatial information gathered at the site. So, it was pointed out the significance and stresses the need for an extremely well-organized data collection system and welldefined ontology as necessary foundations for successful outcomes. This meant that archaeologists should had begun to move toward GIS since other data sources and documentation were moving in that direction. This had as result, the need for improvement of artifact classification at archaeological sites. The interpretation and consistency of reporting currently vary from location to location, and it was proposed semantic and ontological standards for analytical purposes and deeper analytical discovery. Furthermore, it was argued that large repositories of information could be greatly enhanced and more effectively queried with the proposed standardization. Pre-defined choices, well thought out entities, attributes, domains, and relationships were critical foe the success of the Geodatabase.

All the data from the archaeological excavation were coming in a wide variety of formats, sources, and in varying degrees of accuracy. One of the goals was to unite those sources into a single cohesive structure to improve the quality of the data and demonstrate that the data sets can be combined spatially to produce robust visualizations based on a wellorganized framework and classification system. Much of the data received was in tabular format and required some cleanup, preparation, and reestablishing relationships before it could be imported into Esri ArcMap version 10.4.

All the data from the MS Access database tables was easily exported into Excel spreadsheets, while typical entry errors were corrected manually. Additionally, new worksheets were created to mirror the design of the ERD for loading into ArcMap. By using the VLOOKUP feature, all the relevant information related to each fins ID and as a result was the complete record for each artifact. A total of Easting and Northing coordinates from point data collected in the field were entered in order to import the data into ArcMap, resulting in the desired spatial rendering (Fig. 1). Plotted points represent each record after importing to ArcMap [11].

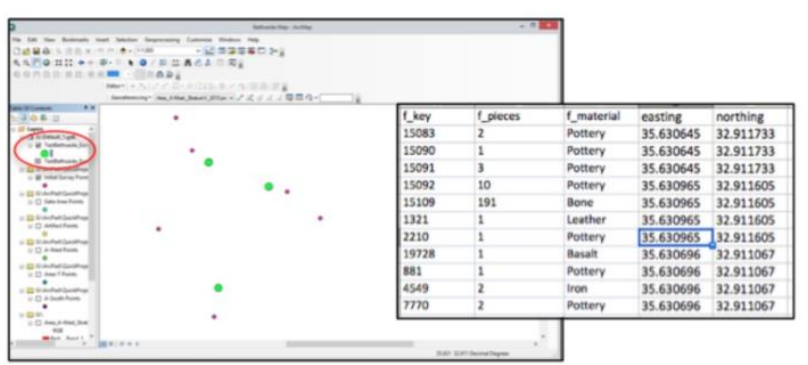

Fig. 1. Sample legacy data (right) successfully imported to ArcMap with resulting points (left). Source: [11].

Detailed CAD drawings (Fig. 2) were used to further confirm accuracy of reference points and monuments. Each was digitizing into JPG format, imported to ArcMap as a data layer, and georeferenced using field collection data points via the georeferencing tool in ArcMap [11].

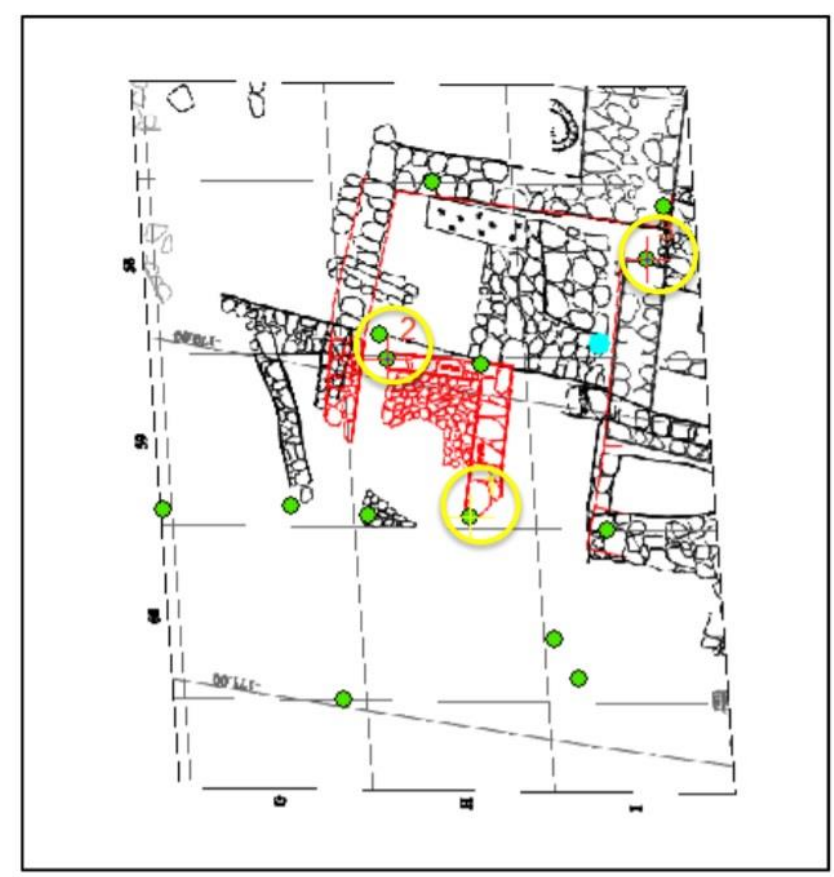

Fig. 2. Imported CAD drawing with 3 georeferenced points. Source: [11].

In the ArcMap software was applied the excavation grid, as the suitable strategy in order to establish consistent reference points in the digital environment. Besides it is known that the grid acts as an overarching framework at the site to provide "constant" reference points when measuring distances and elevations in the field, providing the same time historical monument information and assists in confirming geolocation accuracy of new data. This grid was implemented in the ArcMap, based on the existing data from the database.

Finally, a high resolution aerial photography of the excavation was obtained and added to ArcMap as a data layer. This layer became the basemap for the project (Fig. 3). Point data collected in the field was added to the map as layers, which were then used to geo reference the imagery using the Georeferencing tool in ArcMap software [11]. 


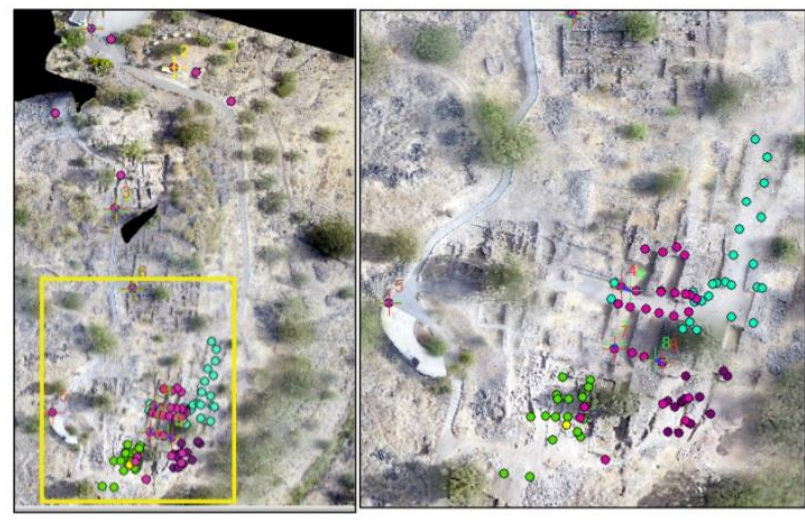

Fig. 3. Excavation overview after Georeferencing. Entire excavation overview (left), and southern end of excavation shown in more detail (right).Source: [11].

To summarize, what was implemented in this program was the application of GIS on existing data from the initial MSAccess database. It was followed the initial design of the database and was added the data that was necessary for Georeferencing. The outcome was the results showed in the base now have their exactly geographical location. So, it could be seen all the artifacts found from a specific time period with their geographical location (Fig. 4), or it could be done a combination of finds from different historical periods (Fig. 5) [11].

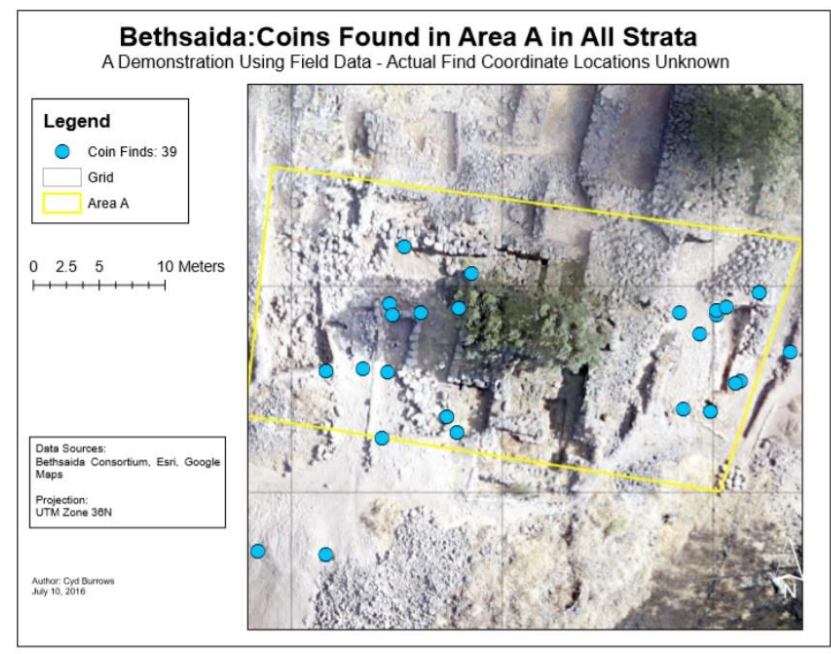

Fig. 4. Variation of visualization of coin data. Source: [11].

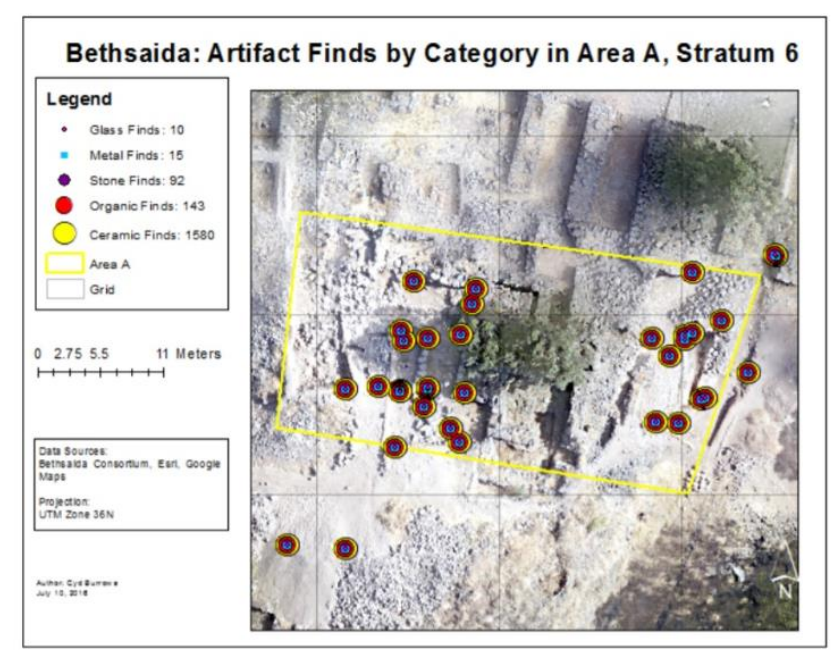

Fig. 5. Visualization of varying categories of finds in an area. Source: [11].
What this project pointed out was that if exists an archaeological database with its data, then it's easy to apply the GIS techniques for combining both, archaeological and Georeferencing data. Due to the nature of some finds, it is extremely important to be able to show the exact extraction point location in order to validate surrounding finds and sites.

\section{CONCLUSION}

The first major activity that must be pre-designed for the simpler operation of any archaeological research and excavation work is the compilation of a database, whether in an analog format as in previous years or in newer digital formats, with the data of each research.

The database should be developed in such a way that it can be updated and changed as the research continues, and the geographical digital databases, as briefly described in this article, play a key role, as the Location tabs are a key building block for any kind of geographic information database.

As is now understood, the database itself is based on parameters that will be set by each creator based on the simplest and most direct access to the findings. Each finding's report is supplemented with bibliographic references, archaeometric data, and any other useful information deemed necessary.

Automatic search, recording, organizing, and updating information, easy data retrieval, representation of geographic information of the data itself, and a variety of other analysis are all available in a modern, integrated digital database for providing archaeological information intelligible both for archaeologists and for the public.

Even through database field correlations, new questions or useful conclusions for archaeological research can emerge, which would be unsatisfactory or impossible to interpret using traditional methods. The analyses and correlations that can be produced using even more modern approaches such as Artificial Intelligence, Machine Learning, and Big Data analysis can provide new data and lead archaeological exploration to new, even more, fascinating paths.

\section{REFERENCES}

[1] Goyolu, C. "Modeling the Archaeological cadastre within internationa standards" Diploma Thesis to School of Rural and Surveying Engineering, Topography and Cadastre Section, National Technical University of Athens, Athens, 2013.

[2] Cholakis, D.N. 'GIS: An introduction", in Data collection, sources and their introduction in GIS. Digital Academy, Athens, 2013.

[3] Nick, R. 'Databases', Internet Archaeology, 28 Jan 2004.

[4] Tripcevich, N. and Wernke, S.A. "On-Site Recording of Excavation Data Using Mobile GIS”, Journal of Field Archaeology, 35:4, 380-397, 2010. DOI: 10.1179/009346910X12707321242511.

[5] Roosevelt, C.H., Cobb, P., Moss, E., Olson B.R. \&Ünlüsoy S "Excavation is Destruction Digitization: Advances in Archaeological Practice", Journal of Field Archaeology, 40:3, 325-346, 2015. DOI: 10.1179/2042458215Y.0000000004.

[6] Malaperdas, G. and Zacharias, N. "A Geospatial Analysis of Mycenaean Habitation Sites Using a Geocumulative versus Habitation Approach". Journal of Geoscience and Environment Protection, 6, 111131, 2018. doi: 10.4236/gep.2018.61008.

[7] Malaperdas, G. \& Zacharias, N. "The habitation Model Trend Calculation (MTC): A new effective tool for predictive modeling in archaeology", Geo-spatial Information Science, 22:4, 314-331, 2019. DOI: 10.1080/10095020.2019.1634320.

[8] Tsipidis, S., Katsianis, M., Kotsakis, K., and Kousoulakou, A. 'Digital technology and excavation: A new approach to the documentation and 
interpretation of archaeological data using Geographic Information Systems' in N.Zacharias (eds) Proceedings of the 2nd ARCH-RNT Symposium, Archaeological Research and New Technologies, University of the Peloponnese, Kalamata, 2012.

[9] González-Tennant, E. 'Using Geodatabases to Generate Living Documents for Archaeology: A Case Study fromthe Otago Goldfields, New Zealand', Historical Archaeology, pp. 20-37, 2009.

[10] Kvamme, K.L. 'Recent Direction and Development in Geographical Information Systems', Journal of Archaeological Research, pp. 153 $202,1999$.
[11] Burrows, C. "Developing an Archaeological Specific Geodatabase to Chronicle Historical Perspectives at Bethsaida, Israel”, USC Graduate School University of Southern California, A Thesis Presented to the Faculty of the USC Graduate School University of Southern California, 2016.

[12] Gonzalez-Tennant, E.M. 'A sample geodatabase structure for managing archaeological data and resources with ArcGIS', Technical Briefs in Historical Archaeology, pp. 12-23, 2007. 